Article

\title{
A Novel QoS Provisioning Algorithm for Optimal Multicast Routing in WMNs
}

\author{
Weijun Yang * and Yuanfeng Chen \\ Department of Electromechanical Engineering, Guangzhou City Polytechnic, Guangzhou 510405, China; \\ cyf@gcp.edu.cn \\ * Correspondence: ywj@gcp.edu.cn; Tel.: +86-020-36248840
}

Academic Editor: Carmen de Pablos Heredero

Received: 13 June 2016; Accepted: 22 July 2016; Published: 1 August 2016

\begin{abstract}
The problem of optimal multicast routing in Wireless Mess Networks (WMNs) with Quality-of-Service (QoS) provisioning, which is Non-Deterministic Polynomial (NP)-complete, is studied in this paper. The existing algorithms are not very efficient or effective. In order to find an approximation optimal solution for WMNs in feasible time from source to the set of destination nodes, combining the previous deterministic algorithm with the well-known Minimum Path Cost Heuristic (MPH) algorithm, a novel multicast heuristic approximation (NMHA) algorithm with QoS provisioning is proposed in this paper to deal with it. The theoretical validations for the proposed algorithm are presented to show its performance and efficiency. After that, the random static networks with different destination nodes are evaluated. Simulations in these networks show that the proposed algorithm can achieve the approximate optimal solution with the approximation factor of $2(1+\varepsilon)(1-1 / q)$ and the time complexity of $\mathrm{O}\left(q m n^{2} \tau^{\mathrm{K}-1}\right)$.
\end{abstract}

Keywords: multicast routing; Wireless Mess Networks; QoS provisioning; approximation algorithm

\section{Introduction}

In a relatively short period of time, the Internet has become a critical information delivery infrastructure for global commerce and multimedia [1]. Over the last decade, multicast routing trees for efficient one-to-many communications applications (e.g., web broadcasting, video teleconferencing, Voice over Internet Protocol (VOIP), and High Definition Television (HDTV) have emerged on the Internet in WMNs [2]. Considering different quality-of-service (QoS) provisioning, one of the solutions is the minimal-cost multicast routing. This presents the problem of finding a tree in a graph from the source to a subset of vertices called terminals such that the total edge cost is minimal. Nevertheless, these days, more and more practical problems, including Very Large Scale Integration (VLSI) layout, network design, etc., can be modeled as multicast routing tree subject to multiple QoS constraints.

The challenge of multicast routing subject to multiple QoS constraints is usually known as the multiply constrained multicast routing (MCMR) problem. To the best of our knowledge, finding the minimal-cost multicast routing tree (or a Steiner tree) is Non-Deterministic Polynomial (NP)-hard [3]. In that way, it is also NP-hard to find the minimal-cost multicast routing tree subject to multiple QoS constrains. On one hand, there has been much work involved in deterministic algorithms [4], such as the genetic algorithm and the ant colony algorithm, which could finally find optimal solutions for this problem, while the time complexity grows exponentially with the increase of network nodes. On the other hand, there also has been much work involved in designing heuristic solutions for this problem [5-13], and they can find multicast trees close to the optimal multicast tree in feasible time, which is more suitable for network multicast use, due to the computing and processing performance of network nodes. 
Recently, it seems that much work has been turned to focus on approximation algorithms to deal with the multiply constrained multicast routing (MCMR) problem. The authors did research on the Steiner tree problem at the earliest, then put forward the MPH algorithm to deal with it [14]. Based on Yuan's study [15], Xue et al. proposed various polynomial time algorithms for the multiply constrained optimal paths (MCOP) problem [16,17]. Feng and Korkmaz introduced a novel idea to show how a heuristic method can be used to boost the average performance of an approximation algorithm [18]. Szymanski presented a Constrained Multicast-Max-Flow-Min-Cost algorithm to maximize the throughput of a multicast tree using network coding, subjected to routing cost constraints [19]. At the same time, the authors did research on the optimal constrained path routing in WMNs in the previous paper [20]; following that, the Fast Minimum Path Cost Heuristic (FMPH) algorithm was put forward to deal with the MCMR problem in WMNs. Recently, Athanasios Vasilakos and his co-authors proposed the cross-layer protocol to decrease the delay [21-25] and devised a novel energy-saving algorithm. Alasaad and his co-authors argued for a ring-based multicast routing topology with support from infrastructure nodes for group communications in WMNs [26], and then developed heuristic algorithms to deal with it. Other excellent research on QoS multicast in wireless networks could be found in this literature [27-35].

Rather than the previous research model, in this paper, multiply constrained optimal multicast routing (MCOMR) is investigated from the perspective of approximation. As far as we know, Xue's algorithm is currently the fastest for path establishment in unicast routing with multiple QoS constraints [16], and the MPH algorithm is efficient for multicast routing [14]. However, the solution to the MCOMR problem is rarely found or is found to be inefficient in the existing literature. In this study, a novel approximation algorithm is proposed to achieve a more excellent solution. The main contributions made in this paper are summarized as follows:

- We present a model for the MCOMR problem from the approximate perspective. This model allows multiple QoS metrics to be considered in WMNs with guaranteed multicast service performance.

- We formulate the problem of the MCOMR problem and develop a novel multicast heuristic approximation (NMHA) algorithm based on the technique of auxiliary graph construction, scaling, rounding and the MPH algorithm to solve the problem.

- We analyze the theoretical properties of the proposed algorithm. Analytical results show that our algorithm is effective and achieves lower complexity and the approximate optimal solution for WMNs.

- We conduct experiments to evaluate the performance of the proposed algorithm and compare the algorithm against variations of current best known algorithms. Obtained numerical results indicate that the proposed algorithm is efficient and accurate for multicast service in WMNs.

The rest of this paper is organized as follows: Section 2 formally formulates the problem to be studied, along with notations, and describes the previous algorithms that will be used in later sections. Section 3 presents the approximation algorithm for the optimal multicast routing with multiple QoS constraints and its theoretical analysis. Simulations obtained from special network are reported to verify the algorithm in Section 4 . Finally, Section 5 summarizes this paper.

\section{Preliminaries}

\subsection{Problem Formulation}

A wireless communication network with $\mathrm{K}$ QoS constraints can be represented by a connected and undirected graph $\mathbf{G}(\mathbf{V}, \mathbf{E}, \overrightarrow{\mathbf{W}}, \overrightarrow{\mathbf{L}})$, where $|\mathbf{V}|=n$ is the set of vertices, $|\mathbf{E}|=m$ is the set of edges, $\overrightarrow{\mathbf{W}}$ is the set of weights, and $\overrightarrow{\mathbf{L}}$ is the set of constraints. Each edge has $K(K \geqslant 2)$ weights, and $\overrightarrow{\mathbf{W}}=\left\{w_{i}\left(e_{x}\right)\right\}, w_{i}\left(e_{x}\right) \geqslant 0$ is the $i$ th weight of an edge $\left(e_{x} \in \mathbf{E}, 1 \leqslant i \leqslant \mathrm{~K}\right)$, a positive constant matrix 
$\overrightarrow{\mathbf{L}}=\left(\mathrm{L}_{1}, \mathrm{~L}_{2}, \ldots, \mathrm{L}_{\mathrm{K}}\right)$ is the $\mathrm{K}$ constraint. Referring to previous related works, the authors have studied the constrained path routing in the literature [20], and then we have the definitions as follows.

Definition 1. MCMR Problem. Consider an undirected graph $\mathbf{G}(\mathbf{V}, \mathbf{E}, \overrightarrow{\mathbf{W}}, \overrightarrow{\mathbf{L}})$. Let $s \in \mathbf{V}$ be the source node and $\mathbf{D}$ be the set of destination nodes, where $\mathbf{D}=\left\{d_{k}\right\} \subseteq \mathbf{V}, s \notin \mathbf{D}, k=1,2, \ldots q$. Denote $\mathbf{T}\left(\mathbf{V}_{\mathbf{T}}, \mathbf{E}_{\mathbf{T}}\right)=\sum_{e_{x} \in \mathbf{T}} e_{x}$ as a multicast routing tree from source $s$ to the set of destination nodes $\mathbf{D}$ in $\mathbf{G}$ with $\mathbf{D} \subseteq \mathbf{V}_{\mathbf{T}} \subseteq \mathbf{V}, \mathbf{E}_{\mathbf{T}} \subseteq \mathbf{E}$. Denote $w_{i}\left(\mathbf{T}_{j}\right)=\sum_{e_{x} \in \mathbf{T}_{j}} w_{i}\left(e_{x}\right), 2 \leqslant i \leqslant$ as the sum of the ith weight on edges along multicast tree $\mathbf{T}_{j}$. The goal is to find a multicast routing tree $\mathbf{T}_{j}$ from $s$ to $\mathbf{D}$, such that $w_{i}\left(\mathbf{T}_{j}\right) \leqslant \mathrm{L}_{i}$. We say that MCMR is feasible if it has a feasible solution; otherwise, it is infeasible.

Definition 2. MCOMR Problem. It is a feasible multicast routing tree for $\mathbf{T}_{j}$, where $w_{i}\left(\mathbf{T}_{j}\right) \leqslant \theta_{j} \mathrm{~L}_{i}$ for all $\theta_{j}, \mathbf{L}_{i} \in(0,1]$. The entire feasible multicast routing tree in $\mathbf{G}(\mathbf{V}, \mathbf{E}, \overrightarrow{\mathbf{W}}, \overrightarrow{\mathbf{L}})$ is denoted as $\left\{\mathbf{T}_{j}\right\}$. The goal is to find an optimal multicast routing $\mathbf{T}^{*}$ among feasible trees $\left\{\mathbf{T}_{j}\right\}$ in $\mathbf{G}$ and $\theta^{*}=\min \left\{\theta_{j}\right\}$ such that $w_{i}\left(\mathbf{T}^{*}\right) \leqslant \theta^{*} \mathrm{~L}_{i}$. We call $\theta^{*}$ the optimal value to MCMR and $\mathbf{T}^{*}$ an optimal multicast routing tree or an optimal solution to MCMR.

Definition 3. (a-Approximation algorithm). Let $a \geqslant 1$ be a constant. If the algorithm generates a multicast routing tree $\mathbf{T}^{\text {opt }}$ from $s$ to $\mathbf{D}$ such that $w_{i}\left(\mathbf{T}^{\mathrm{opt}}\right) \leqslant a \theta^{*} \mathrm{~L}_{i}, 2 \leqslant i \leqslant \mathrm{~K}$, then $\mathbf{T}^{\text {opt }}$ is called an $a$-Approximation to MCOMR, and it is called an $a$-Approximation algorithm.

Table 1 lists frequently used notations.

Table 1. Frequently used notations.

\begin{tabular}{|c|c|}
\hline$s$ & the source node \\
\hline$d_{k}$ & the $k$ th destination nodes \\
\hline D & the set of destination nodes \\
\hline$m, n$ & the edge and the node number of graph $\mathbf{G}$, respectively \\
\hline$q$ & the number of terminal nodes \\
\hline$w_{i}\left(e_{x}\right)$ & $i$ th link weight of edge $e_{x}$ \\
\hline K & the number of Quality-of-Service (QoS) requirements \\
\hline $\overrightarrow{\mathbf{W}}$ & the set of weights of the edges \\
\hline $\overrightarrow{\mathrm{L}}$ & the set of constrains \\
\hline $\mathrm{L}_{i}$ & $i$ th QoS requirement \\
\hline $\mathrm{T}$ & the multicast tree with all nodes in the set of destination nodes $\mathbf{D}$ \\
\hline $\mathbf{T}_{j}$ & the Steiner tree with $j$ destination nodes in the set $\mathbf{D}$ \\
\hline $\begin{array}{l}\mathbf{V}_{\mathbf{T}}, \mathbf{E}_{\mathbf{T}} \\
\mathbf{T}^{*}, \mathbf{T}^{o p t}\end{array}$ & $\begin{array}{l}\text { all the nodes and the edges of the multicast trees } T \text {, respectively } \\
\text { the optimal and the approximate optimal multicast routing tree, respectively }\end{array}$ \\
\hline$w\left(d_{k}, \mathbf{T}_{j-1}\right)$ & the total weights from $d_{k}$ to $\mathbf{T}_{j-1}$ \\
\hline $\operatorname{PATH}\left(d_{k}, \mathbf{T}_{j-1}\right)$ & the short path connected node $d_{k}$ to the $\mathbf{T}_{j-1}$ \\
\hline $\begin{array}{c}\mathrm{C}_{i}, \mathrm{D}_{i} \\
\mathbf{P}\left(u, \mathrm{C}_{i}, \ldots, \mathrm{C}_{\mathrm{K}}\right)\end{array}$ & $\begin{array}{l}\text { the } i \text { th weight of path length from source } s \text { to any node } u \text { in } \mathrm{G}^{\mathrm{K}, \tau} \\
\text { all the } \mathrm{K} \text { weight of path length from source } s \text { to any node } u \text { in } \mathrm{G}^{\mathrm{K}, \tau}\end{array}$ \\
\hline
\end{tabular}

\subsection{Deterministic Algorithm with Auxiliary Graph}

Xue et al. provided an important methodology for finding the approximate optimal path for constrained unicast routing by constructing an auxiliary graph [16]. It transforms an undirected graph $\mathbf{G}$ to a directed graph $\mathbf{G}^{\mathrm{K}, \tau}$. Each vertex $v \in \mathbf{G}$ is associated with $(1+\tau)^{\mathrm{K}-1}$ vertices, since each weight has been normalized $w_{i}(e) / \mathrm{L}_{i}$. Then, $\tau=\lceil(n-1) / \varepsilon\rceil$ ( $\varepsilon$ is the approximation factor) could be regarded as the maximum integer of all constraints in $\mathrm{G}^{\mathrm{K}, \tau}$. For example, from the source node $s$ to any node $u, C_{i} \in[0, \tau], \forall 2 \leqslant i \leqslant \mathrm{~K}$ is used for recording the $i$ th weight of path length, and $\mathbf{P}\left(u, C_{2}, C_{i}, \ldots, C_{K}\right)$ for all the $K$ weight of path length. To each undirected edge $(u, v)$ in $\mathbf{G}$, the directed edge in $\mathbf{G}^{K, \tau}$ is constructed from $\mathbf{P}\left(u, C_{2}, C_{i}, \ldots, C_{K}\right)$ to $\mathbf{P}\left(v, D_{2}, D_{i}, \ldots, D_{k}\right)$, where $D_{i}=C_{i}+w_{i}(u, v), \forall C_{i}, D_{i} \in[0, \tau]$. It is clear that the infeasible paths of the latter $\mathrm{K}-1$ metrics have been filtered. Therefore, an optimal path in $\mathbf{G}$ corresponds to an optimal path from $\mathbf{P}(s, 0, \ldots, 0)$ to $\mathbf{P}(d, \tau, \ldots, \tau)$ in $\mathbf{G}^{\mathrm{K}, \tau}$. 
The basic idea behind Xue's algorithm is to replace time with space. As far as we know, Xue's approximation scheme with the time complexity of $\mathrm{O}\left(m \tau^{\mathrm{K}-1}\right)$ is currently the fastest way to calculate a path with multiple QoS constraints. As can be observed from the examples, using Xue's deterministic algorithm with an auxiliary graph, it is feasible and meaningful to seek an effective and efficient algorithm to approximate optimal multicast routing with multiple constraints.

\subsection{Description of MPH Algorithm}

In 1980, Takahashi and Matsuyama put forward the MPH algorithm to help solve the Steiner tree problem [14]. The following are its steps:

Step 1: Choose any node $d_{1}$ from the set $\mathbf{D}$ of multicast destination nodes, let $j=1$, and initialize the generation of tree $\mathbf{T}_{1}=\left\{d_{1}\right\}, \mathbf{V}_{\mathbf{T}_{1}}=\left\{d_{1}\right\}$;

Step 2: The cost $w\left(d_{k}, \mathbf{T}_{j-1}\right)$ from $d_{k}(k \leqslant q)$ in $\overline{\mathbf{D} \cap \mathbf{T}_{j-1}}$ to $\mathbf{T}_{j-1}$ can reach the minimum value by comparing formula (1):

$$
w\left(d_{j}, \mathbf{T}_{j-1}\right)=\min \left\{w\left(d_{k}, \mathbf{T}_{j-1}\right) \mid d_{k} \in \overline{\mathbf{D} \cap \mathbf{T}_{j-1}}\right\}
$$

By connecting $d_{j}$ to $\mathbf{T}_{j-1}$ through the shortest $\operatorname{PATH}\left(d_{j}, \mathbf{T}_{j-1}\right)$, the updated tree can get the result of $\mathbf{T}_{j}=\operatorname{PATH}\left(d_{j}, \mathbf{T}_{j-1}\right) \cup \mathbf{T}_{j-1}$;

Step 3: Until $i>q$, find the Steiner generating tree; otherwise, let $j=j+1$, then repeat Step 2.

\section{The Proposed NMHA Algorithm}

\subsection{Description of NMHA Algorithm}

According to the algorithm analysis above, it is concluded that Xue's deterministic algorithm takes the lead in finding the optimal path for the unicast routing, and the MPH algorithm is fit for multicast routing problem subjected to one QoS constraint. Therefore, combining Xue's deterministic algorithm with the MPH algorithm, the NMHA algorithm is presented in this paper to deal with MCOMR problem. The following are its steps:

Step 1: Convert the graph $\mathbf{G}(\mathbf{V}, \mathbf{E}, \overrightarrow{\mathbf{W}}, \overrightarrow{\mathbf{L}})$ to the new graph $\mathbf{G}^{\mathrm{N}}\left(\mathbf{V}, \mathbf{E}, \mathbf{W}^{\mathrm{N}}, \tau\right)$ based on the technique of the scaling and rounding;

Step 2: Convert the graph $G^{N}\left(V, E, \mathbf{W}^{N}, \tau\right)$ to the new graph $G_{K}^{N}\left(V_{K}^{N}, E_{K}^{N}, \mathbf{W}_{K}^{N}, \tau\right)$ based on the technique of the auxiliary graph construction;

Step 3: Choose any node $d_{1}$ from the set $\mathbf{D}$ of multicast destination nodes, let $j=1$, and initialize the generation of tree $\mathbf{T}_{0}{ }^{\text {opt }}=\{s\}, \mathbf{V}_{\mathbf{T}_{0}}=\{s\}$;

Step 4: Calculate the cost $w_{1}\left(d_{k}, \mathbf{T}_{j-1}^{\text {opt }}\right)$ from $d_{k}(k \leqslant q)$ in $\overline{\mathbf{D} \cap \mathbf{T}_{j-1}^{\text {opt }}}$ to $\mathbf{T}_{j-1}^{\text {opt }}$ for all nodes $d_{k}(k \leqslant q)$ by Xue's deterministic algorithm;

Step 5: It can reach the minimum value by comparing formula (2):

$$
w_{1}\left(d_{j}, \mathbf{T}_{j-1}^{\mathrm{opt}}\right)=\min \left\{w_{1}\left(d_{k}, \mathbf{T}_{j-1}^{\mathrm{opt}}\right) \mid d_{k} \in \overline{\mathbf{D} \cap \mathbf{T}_{j-1}^{\mathrm{opt}}}\right\}
$$

By connecting the node $d_{j}$ to $\mathbf{T}_{j-1}^{\mathrm{opt}}$ through the shortest PATH $\left(d_{j}, \mathbf{T}_{j-1}^{\mathrm{opt}}\right)$, the updated tree can get the result of $\mathbf{T}_{j}^{\text {opt }}=\operatorname{PATH}\left(d_{j}, \mathbf{T}_{j-1}^{\text {opt }}\right) \cup \mathbf{T}_{j-1}^{\text {opt }}$; until $i>q$, go to Step 4;

Step 6: Until $i>q$, go to Step 7; otherwise, let $j=j+1$, then repeat Step 5;

Step 7: If $w_{1}^{\mathrm{N}}\left(\mathbf{T}_{j-1}^{o p t}\right)<\tau$, find the Steiner generating tree; Else No feasible multicast routing tree.

\subsection{The Procedure of the NMHA Alogorithm}

The detailed procedure of NMHA is presented as shown in Algorithm 1. 


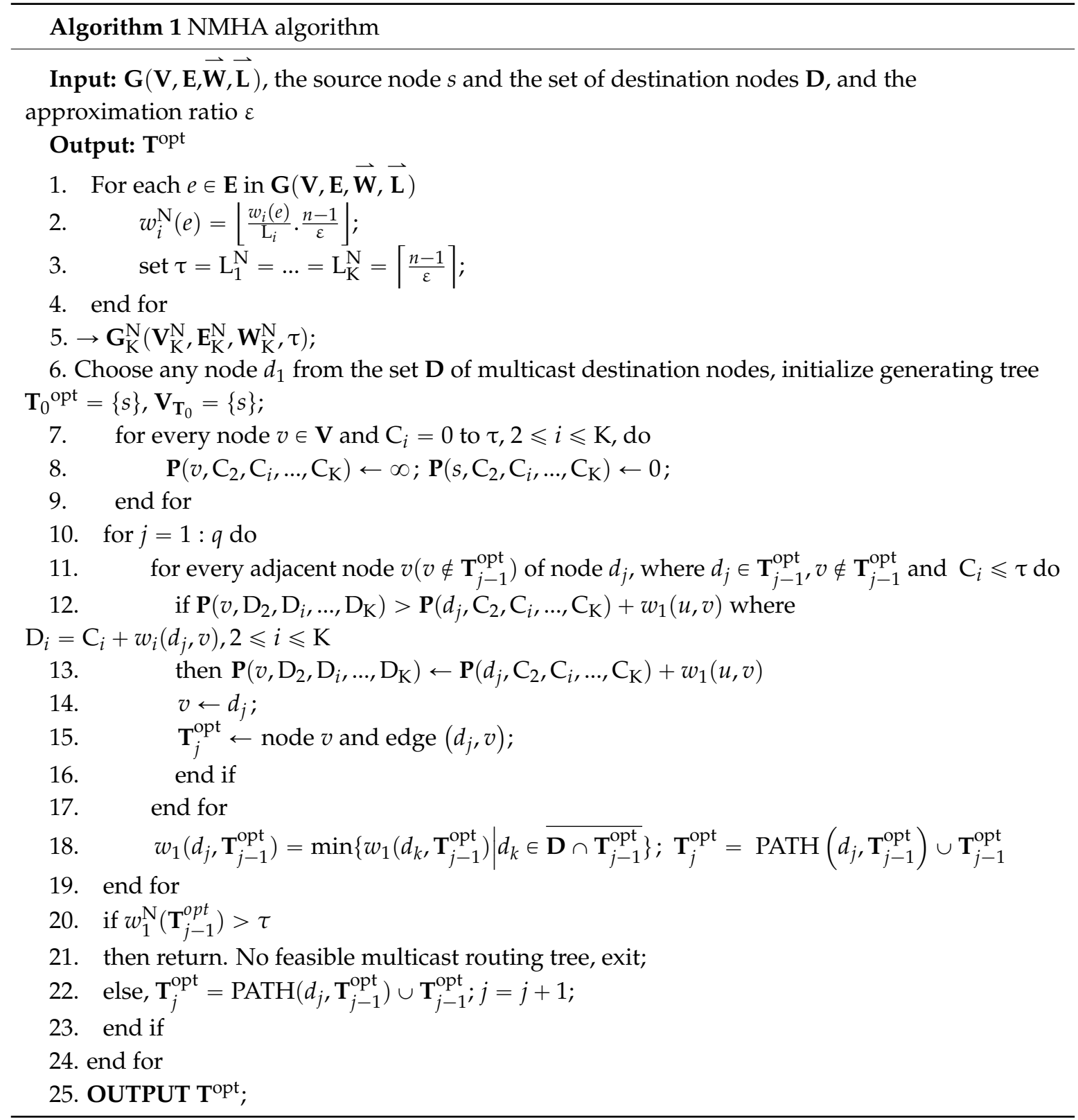

\subsection{Analysis of the NMHA Algorithm}

Theorem 1. NMHA obtains a feasible multicast routing $\mathbf{T}^{\text {opt }}$ from source s to destination set $\mathbf{D}$, which minimizes $\max _{1 \leqslant i \leqslant \mathrm{~K}} w_{i}^{\mathrm{N}}\left(\mathbf{T}_{j}\right)$ among all multicast routing trees in $\mathbf{G}_{\mathrm{K}}^{\mathrm{N}}\left(\mathbf{V}_{\mathrm{K}}^{\mathrm{N}}, \mathbf{E}_{\mathrm{K}}^{\mathrm{N}}, \mathbf{W}_{\mathrm{K}}^{\mathrm{N}}, \tau\right)$. The worst case time complexity of the algorithm is $O\left(q m n^{2} \tau^{\mathrm{K}-1}\right)$.

Proof of Theorem 1. NMHA consists of two major phases. In the first phase, it calculates the value after scaling and rounding for each edge and initializes the data structures (Lines 1-9), the time complexity of which is constant. In the second phase, it computes the optimal multicast routing tree (Lines 10-25), the time complexity of which is a crucial factor. Since it searches $\mathbf{P}\left(v, \mathrm{D}_{2}, \mathrm{D}_{i}, \ldots, \mathrm{D}_{\mathrm{K}}\right)$ in lexicographic order for every adjacent node $v\left(v \notin \mathbf{T}_{j-1}^{\text {opt }}\right)$ of node $d_{j} \in \mathbf{T}_{j-1}^{\text {opt }}$ in the NMHA algorithm (Lines 10-19), and guarantees that $d_{i}$ is not more than the largest integer $\tau$, which means that NMHA searches a shortest path in the space of $\max _{1 \leqslant i \leqslant K} w_{i}^{\mathrm{N}}\left(\mathbf{T}_{j}\right)$, the NMHA obtains a multicast routing tree $\mathbf{T}^{\text {opt }}$ from source $s$ to destination set $\mathbf{D}$ that minimizes $\max _{1 \leqslant i \leqslant K} w_{i}^{\mathrm{N}}\left(\mathbf{T}_{j}\right)$ among all multicast routing trees in $\mathrm{G}_{\mathrm{K}}^{\mathrm{N}}$.

During the running of NMHA, $\mathrm{G}_{\mathrm{K}}^{\mathrm{N}}$ have $\mathrm{O}\left(n \tau^{\mathrm{k}-1}\right)$ vertices and $\mathrm{O}\left(2 m \tau^{\mathrm{k}-1}+n \tau^{\mathrm{k}-1}\right)$ edges, which confirm our observation that the space complexity is fairly large. Moreover, $\mathrm{G}_{\mathrm{K}}^{\mathrm{N}}$ contains a directed 
edge from $\mathbf{P}\left(d_{j}, \mathrm{C}_{2}, \mathrm{C}_{i}, \ldots, \mathrm{C}_{\mathrm{K}}\right)$ to $\mathbf{P}\left(v, \mathrm{D}_{2}, \mathrm{D}_{i}, \ldots, \mathrm{D}_{\mathrm{K}}\right)$ with length of $w_{i}\left(d_{j}, v\right)$. Therefore, the optimal multicast routing tree $\mathbf{T}^{\mathrm{opt}}$ found in NMHA minimizes $\max _{1 \leqslant i \leqslant \mathrm{~K}} w_{i}^{\mathrm{N}}\left(\mathbf{T}_{j}^{\mathrm{opt}}\right)$ among multicast routing trees in $\mathrm{G}_{\mathrm{K}}^{\mathrm{N}}$.

Since each $w_{i}(e)$ is a positive real-value for $2 \leqslant i \leqslant \mathrm{~K}, e \in \mathbf{E}$, the existence of a directed edge from $\mathbf{P}\left(d_{j}, \mathrm{C}_{2}, \mathrm{C}_{i}, \ldots, \mathrm{C}_{\mathrm{K}}\right)$ to $\mathbf{P}\left(v, \mathrm{D}_{2}, \mathrm{D}_{i}, \ldots, \mathrm{D}_{\mathrm{K}}\right)$ in $\mathrm{G}_{\mathrm{K}}^{\mathrm{N}}$ implies that $\mathrm{C}_{i} \leqslant \mathrm{D}_{i}$ for $2 \leqslant i \leqslant \mathrm{~K}, e \in \mathbf{E}$. Therefore, the graph $\mathrm{G}_{\mathrm{K}}^{\mathrm{N}}$ is acyclic. For an acyclic graph, the worst-case time complexity of each running process is (Lines 11-17). As far as we know, the time complexity of the MPH algorithm is $\mathrm{O}\left(q n^{2}\right)$ (Lines 18). Therefore, the worst case time complexity of NMHA is $O\left(q m n^{2} \tau^{\mathrm{K}-1}\right)$.

Theorem 2. To any feasible multicast routing tree $\mathbf{T}_{j}$ in $\mathbf{G}$, it is a feasible multicast routing tree in $\mathbf{G}_{K}^{N}$.

Proof of Theorem 2. To a feasible multicast routing tree $\mathbf{T}_{j}$ in $\mathbf{G}$, it has

$$
w_{i}\left(\mathbf{T}_{j}\right)=\sum_{e \in \mathbf{T}_{j}} w_{i}(e) \leqslant \mathrm{L}_{i}
$$

It implies that

$$
\frac{\sum_{e \in \mathbf{T}_{j}} w_{i}(e)}{\mathrm{L}_{i}} \cdot \beta=\sum_{e \in \mathbf{T}_{j}} \frac{w_{i}(e)}{\mathrm{L}_{i}} \cdot \beta \leqslant \beta
$$

Since

$$
w_{i}^{\mathrm{N}}(e)=\left\lfloor\frac{w_{i}(e)}{\mathrm{L}_{i}} \cdot \beta\right\rfloor \leqslant \frac{w_{i}(e)}{\mathrm{L}_{i}} \cdot \beta
$$

and

$$
\beta \leqslant\lceil\beta\rceil=\tau
$$

Then, it has

$$
\begin{aligned}
w_{i}^{\mathrm{N}}\left(\mathbf{T}_{j}\right) & =\sum_{e \in \mathbf{T}_{j}} w_{i}^{\mathrm{N}}(e) \\
& =\sum_{e \in \mathbf{T}_{j}}\left\lfloor\frac{w_{i}(e)}{L_{i}} \cdot \beta\right\rfloor \\
& \leqslant \sum_{e \in \mathbf{T}_{j}} \frac{w_{i}(e)}{L_{i}} \cdot \beta
\end{aligned}
$$

According to Equations (4), (6) and (7), it has

$$
w_{i}^{\mathrm{N}}\left(\mathbf{T}_{j}\right) \leqslant \tau
$$

Hence, any feasible multicast routing tree $\mathbf{T}_{j}$ in $\mathbf{G}$ is a feasible multicast routing tree in $\mathbf{G}_{\mathrm{K}}^{\mathrm{N}}$.

Theorem 3. The NMHA algorithm finds a $2(1+\varepsilon)(1-1 / q)$-approximation multicast routing tree to MCOMR.

Proof of Theorem 3. To the optimal multicast routing tree $\mathbf{T}^{*}$ in $\mathrm{G}$, it has

$$
\begin{gathered}
w_{i}\left(\mathbf{T}^{*}\right) \leqslant \theta^{*} \cdot \mathrm{L}_{i} \\
w_{i}^{\mathrm{N}}\left(\mathbf{T}^{*}\right)=\sum_{e \in \mathbf{T}^{*}} w_{i}^{\mathrm{N}}(e)=\sum_{e \in \mathbf{T}^{*}}\left\lfloor\frac{w_{i}(e)}{\mathrm{L}_{i}} \cdot \beta\right\rfloor
\end{gathered}
$$

This implies that

$$
\begin{aligned}
w_{i}^{\mathrm{N}}\left(\mathbf{T}^{*}\right) & \leqslant \sum_{\substack{e \in \mathbf{T}^{*} \\
w}}\left(\frac{w_{i}(e)}{\mathrm{L}_{i}} \cdot \beta\right) \\
& =\frac{w_{i}\left(\mathbf{T}^{*}\right)}{\mathrm{L}_{i}} \cdot \beta \\
& \leqslant \theta^{*} \cdot \beta
\end{aligned}
$$


On the other hand, $\mathbf{T}^{\text {opt }}$ minimizes $\max _{1 \leqslant i \leqslant K} w_{i}^{\mathrm{N}}\left(\mathbf{T}_{j}\right)$ among all multicast routing trees in $\mathbf{G}_{\mathrm{K}}^{\mathrm{N}}$ and $\mathbf{T}^{*}$ is a feasible multicast routing tree as mentioned in Theorem 1 and in Theorem 2, respectively, then it has

$$
\begin{aligned}
\max _{1 \leqslant i \leqslant \mathrm{~K}} w_{i}^{\mathrm{N}}\left(\mathbf{T}^{\mathrm{opt}}\right) \leqslant & \max _{1 \leqslant i \leqslant \mathrm{~K}} w_{i}^{\mathrm{N}}\left(\mathbf{T}^{*}\right) \\
w_{i}^{\mathrm{N}}\left(\mathbf{T}^{\text {opt }}\right)=\sum_{e \in \mathbf{T}^{\text {opt }}} w_{i}^{\mathrm{N}}(e) & =\sum_{e \in \mathbf{T}^{\text {opt }}}\left\lfloor\frac{w_{i}(e)}{\mathrm{L}_{i}} \cdot \beta\right\rfloor \\
& \geqslant \sum_{e \in \mathbf{T}^{\text {opt }}}\left(\frac{w_{i}(e)}{\mathrm{L}_{i}} \cdot \beta-1\right)
\end{aligned}
$$

Since $\mathbf{T}^{\text {opt }}$ have $|\mathbf{V}|-1$ hops at most, and there are $|\mathbf{V}|=n$ nodes in $\mathbf{G}$, it implies that

$$
\begin{aligned}
\sum_{e \in \mathbf{T}^{\text {opt }}}\left(\frac{w_{i}(e)}{\mathrm{L}_{i}} \cdot \beta-1\right) & =\sum_{e \in \mathbf{T}^{\text {opt }}}\left(\frac{w_{i}(e)}{\mathrm{L}_{i}} \cdot \beta\right)-\sum_{e \in \mathbf{T}^{\text {opt }}} 1 \\
& \geqslant \frac{w_{i}\left(\mathbf{T}^{\text {opt }}\right)}{\mathrm{L}_{i}} \cdot \beta-(n-1)
\end{aligned}
$$

According to Equations (11), (12) and (14), it has

$$
\frac{w_{i}\left(\mathbf{T}^{o p t}\right)}{\mathrm{L}_{i}} \cdot \beta-(n-1) \leqslant \theta^{*} \cdot \beta
$$

It implies that

$$
\frac{w_{i}\left(\mathbf{T}^{o p t}\right)}{\mathrm{L}_{i}} \leqslant \theta^{*}+\frac{n-1}{\beta}
$$

Let $\frac{n-1}{\beta}=\varepsilon \cdot \theta^{*}, \varepsilon>0$, then

$$
w_{i}\left(\mathbf{T}^{o p t}\right) \leqslant(1+\varepsilon) \cdot \theta^{*} \cdot \mathrm{L}_{i}, 1 \leqslant i \leqslant \mathrm{~K}
$$

Takahashi and Matsuyarna have proved in literature [14] that there is corresponding relationship between each $\mathrm{j}$ and nodes pair $\left(t_{j-1}, t_{j}\right)$, in which $i, j=2,3, \ldots, q$ and $v_{1}, v_{2}, \ldots, v_{\mathrm{k}}$ are from 1 to $\mathrm{k}$. Let $j$ and number pair $\left[t_{q(j)-1}, t_{q(j)}\right]$ be one-to-one relationship:

$$
\begin{aligned}
w_{i}\left(\mathbf{T}_{\mathrm{MPH}}^{\mathrm{opt}}\right) & =\sum_{i=2}^{q} c\left(v_{i}, \mathbf{V}_{i-1}\right) \leqslant \sum_{i=2}^{q} c\left(v_{t_{q(i)-1}}, v_{t_{q(i)}}\right) \\
& =\sum_{j=2}^{q} c\left(v_{t_{j-1}}, v_{t_{j}}\right) \leqslant 2(1-1 / q) \cdot w_{i}\left(\mathbf{T}^{*}\right)
\end{aligned}
$$

According to Equations (17) and (18), it has

$$
w_{i}\left(\mathbf{T}_{N M H A}^{o p t}\right)=w_{i}\left(\mathbf{T}_{M P H}^{o p t}\right) \leqslant 2(1+\varepsilon)(1-1 / \mathbf{q}) \cdot \theta^{*} \cdot \mathrm{L}_{i}, 1 \leqslant i \leqslant \mathrm{~K}
$$

Hence, the NMHA algorithm can finally find a $2(1+\varepsilon)(1-1 / q)$-approximation multicast routing tree to MCOMR, and the time complexity of the algorithm is $O\left(q m n^{2} \tau^{\mathrm{K}-1}\right)$.

Theorem 4. The NMHA algorithm is a sub-optimal algorithm for the MCOMR problem, and the upper bound of approximation ratio is $1+\varepsilon$.

Proof of Theorem 4. The authors in the literature [35] have shown that the total MOS problem was equivalent to generalize maximum coverage (GMC) problem, and then proved that for any $\varepsilon>0$, it had no $(1-1 / e+\varepsilon)$ approximation algorithm unless $\mathbf{P}=\mathbf{N P}$. The approximation algorithm NMHA proposed in this paper for the MCOMR problem is the same as that in [35]. It has been proven in Theorem 3 that the NMHA algorithm can finally find a $2(1+\varepsilon)(1-1 / q)$-approximation multicast 
routing tree to MCOMR. Thus, the upper bound of approximation ration should be discussed to evaluate the performance of NMHA. To the optimal multicast routing tree $\mathbf{T}^{*}$ in $\mathbf{G}$, it has

$$
w_{i}\left(\mathbf{T}^{*}\right) \leqslant \theta^{*} \cdot \mathrm{L}_{i}, 1 \leqslant i \leqslant \mathrm{~K}
$$

According to Equations (19) and (20), it has

$$
w_{i}\left(\mathbf{T}_{N M H A}^{o p t}\right) \leqslant 2(1+\varepsilon)(1-1 / q) \cdot w_{i}^{*}, \text { s.t. } w_{i}^{*}=\theta^{*} \cdot \mathrm{L}_{i}
$$

The multicast routing is discussed in this paper, which means that number of destination nodes are more than two, it has

$$
2(1+\varepsilon)(1-1 / q) \leqslant 2(1+\varepsilon) \cdot \frac{1}{2}=1+\varepsilon
$$

Hence, the upper bound of approximation ration of the NMHA algorithm is $1+\varepsilon$, and thus the theorem is proven.

\section{The Simulation Experiments}

This section shall evaluate both the performance of NMHA and the performance of an experimentally obtained multicast routing tree. To evaluate its efficiency, NMHA is implemented and conducted in a performance study.

An example of the NMHA algorithm is shown in Figure 1. Figure 1a is a simple topology graph G of a static undirected network, in which the nodes in blue are the set of destination nodes $\mathbf{D}$ and others are non-multicast destination nodes. The numbers marked between two nodes are linked to cost or distance. The node is originally added to the generating tree $T_{0}=\{\mathrm{A}\}$. In order to find the multicast generating tree with the NMHA algorithm (Lines 10-11), the first node $U_{1}$ is selected, and then it is added to the tree A- $U_{1}$ in Figure $1 b$ (Lines 12-20) with the total cost of the generating tree 3 . In the following phase of the algorithm, destination nodes $U_{2}$ and $U_{3}$ are added to the tree, respectively, and the multicast destination nodes are sorted as $\mathrm{U}_{1}-\mathrm{U}_{2}-\mathrm{U}_{3}$. The final multicast tree obtained, whose total cost is 7, is shown in Figure 1c. According to the NMHA algorithm proposed in this paper, the generating graph is found.

An Novel Multicast Heuristic Approximation Algorithm in Wireless Mesh Networks

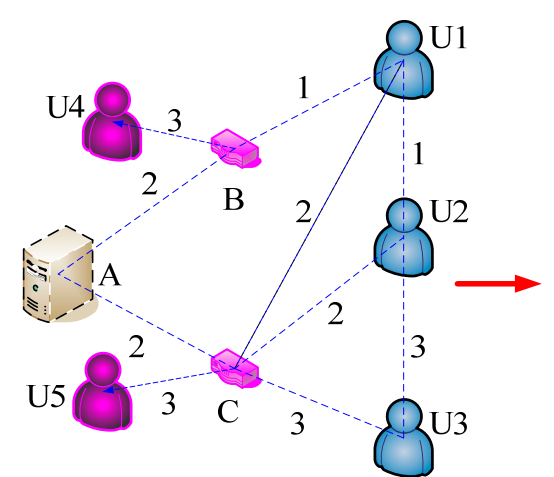

(a) step 1 of NMHA

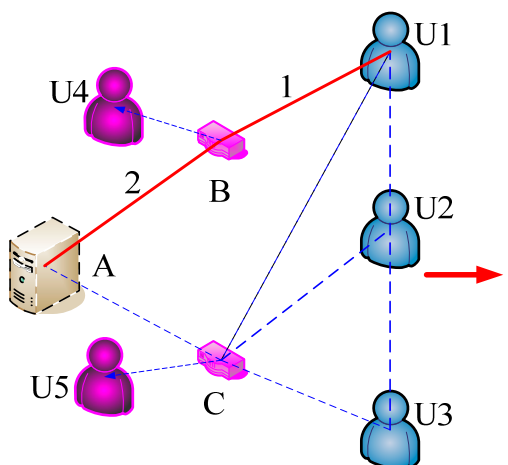

(b) step 2 of NMHA

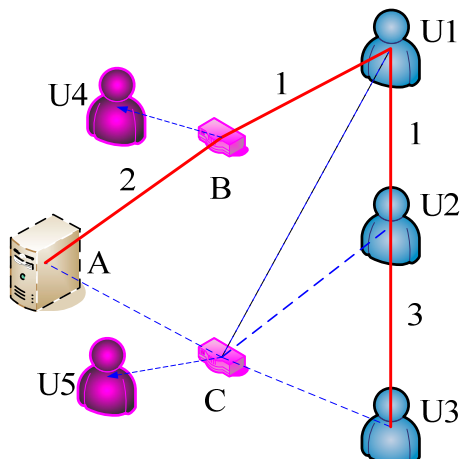

(c) step 3 of NMHA

Figure 1. An example of the novel multicast heuristic approximation (NMHA) algorithm. (a), (b) and (c) are the step 1, step 2 and step 3 of NMHA, respectively.

To examine the performance of NMHA, we use the famous Waxman model to generate a large-size Random Network (RNET). The random static network RNET, is used in these experiments, which runs on an Intel Core Duo CPU $1.66 \mathrm{GHz}$ PC with 2 GB memory (Lenovo, Beijing, China). There are 60 nodes and 100 edges in RNET as shown in Figure 2, and other parameters for the network were 
produced randomly by Waxman model. Each link of RNET has three weights, which correspond to Cost, Delay and Jitter.

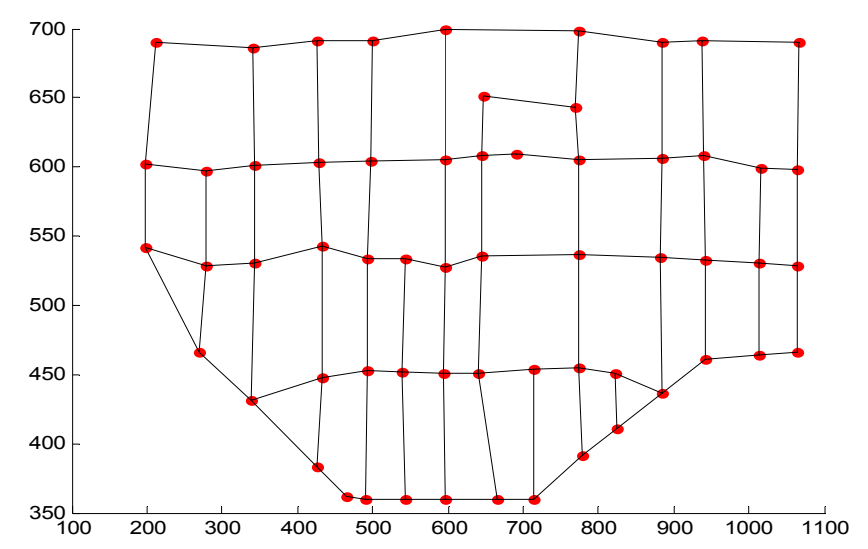

Figure 2. The network topology of Random Network (RNET).

All the red points denote the nodes of the network, the black wires denote the links, the green circles denote the source node $s$ (No. 1) and the green stars denote the set of destination nodes $\mathbf{D}$ (No. 30, 51 and 55) in the network RNET, which is shown in Figure 3. Comparison with the costs from the source node $s$ to every destination node by Xue's deterministic algorithm, the minimal-cost node (No. 30) is selected first. Figure 4 shows the minimal-cost path from source node (No. 1) to destination nodes (No. 30), which is selected first by NMHA in the network. The blue paths in these figures indicate the minimal or the optimal paths from source to destination. Figure 5 illustrates that the following node (No. 55) is added to the tree by NMHA. As expected, the optimal multicast routing tree with three constraints added by the algorithm NMHA can be found after the last node (No. 51) is added to the multicast tree. At last, the optimal multicast routing tree described in Figure 6 shows the corresponding multicast tree from the source node (No. 1) to the set of destination nodes $\mathbf{D}$ (No. 30, 51 and 55).

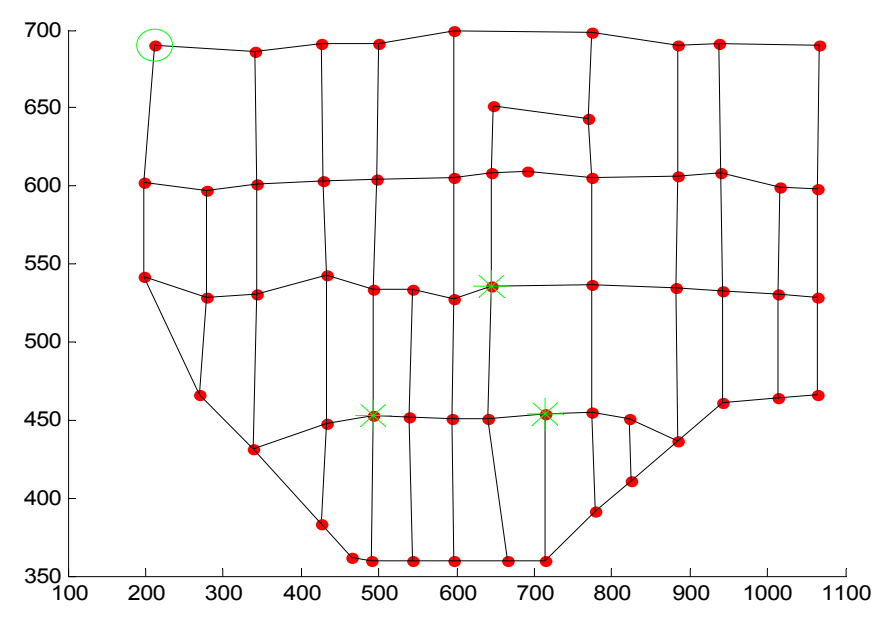

Figure 3. The multicast destination nodes. 


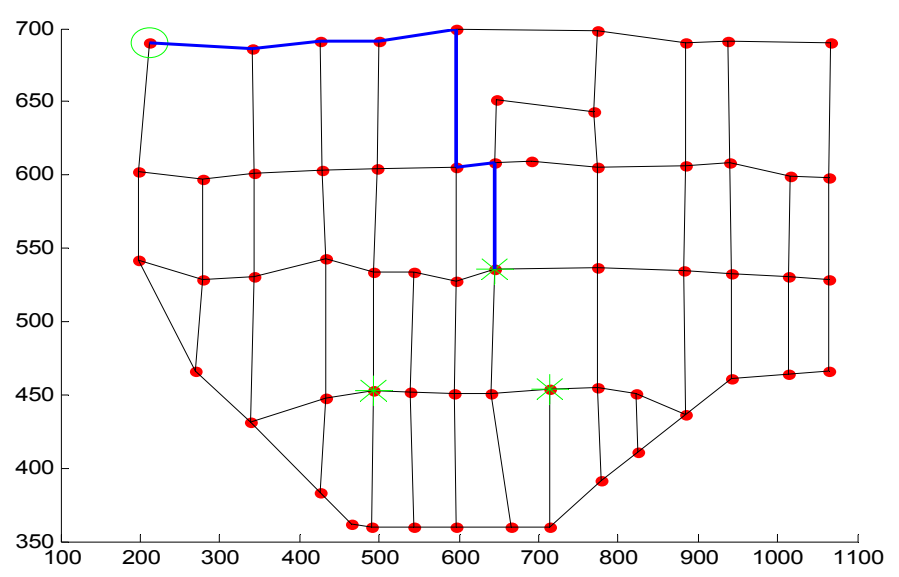

Figure 4. The No. 30 node connected to the multicast tree.

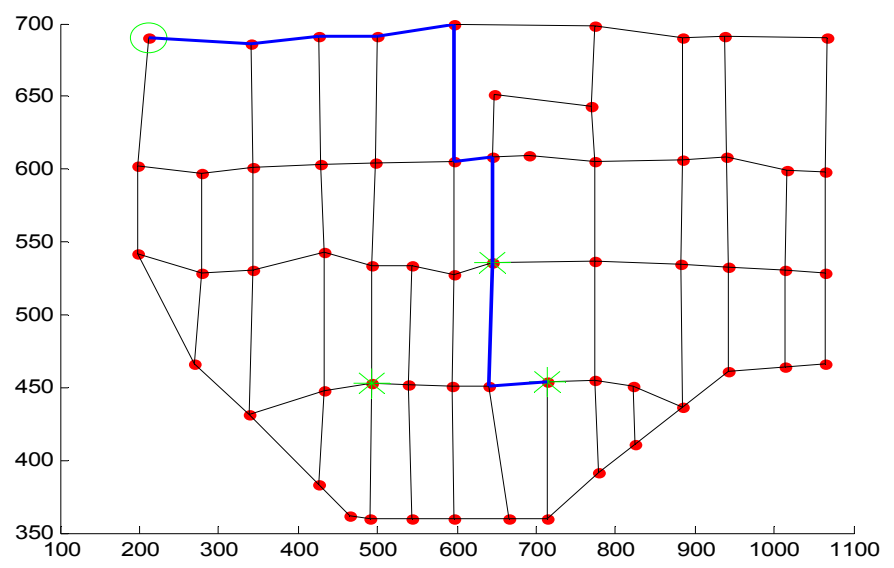

Figure 5. The No. 55 node connected to the multicast tree.

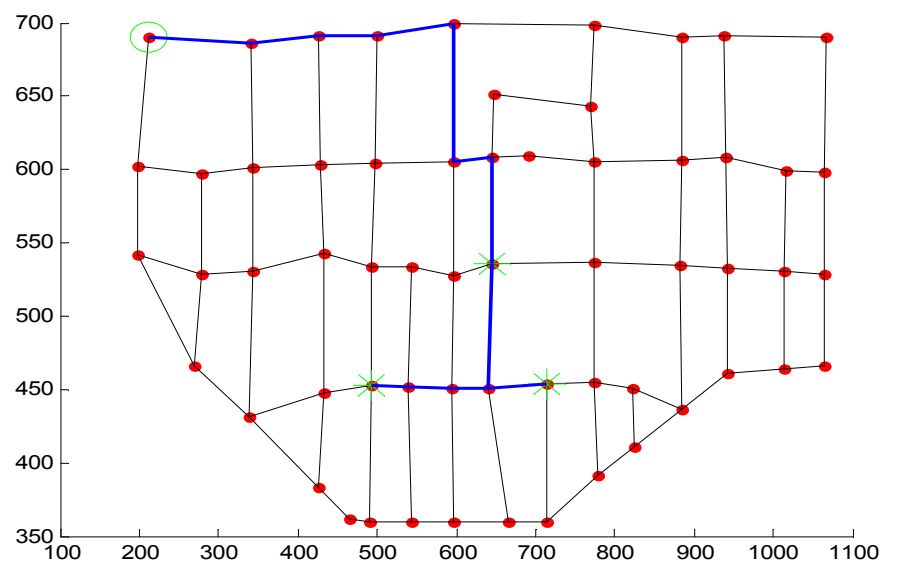

Figure 6. The No. 51 node connected to the multicast tree.

In these simulation experiments, the approximating Steiner tree could be found by the NMHA algorithm presented in this paper. Following that the efficiency of the NMHA algorithm would be verified. Table 2 shows the comparisons of average time consuming for Steiner trees (ATCS) generated via the NMHA to Xue's deterministic algorithm in the simulation experiments, respectively. With the two algorithms, q destination nodes in the RNET network are randomly generated, and then the Steiner trees are obtained and the corresponding times are recorded. The simulations experiments are 
made to calculate the average values for ten times. In order to evaluate the efficiency of two algorithms, we define the following metrics:

$$
\text { ATCS }=\frac{\text { Total time consuming for each Steiner tree with q destination nodes }}{\text { Number of running }}
$$

Table 2. A comparison of the performance of novel multicast heuristic approximation (NMHA) algorithm and Xue's algorithm [16] in the random network.

\begin{tabular}{ccccccccc}
\hline NO. & $q=2$ & $q=5$ & $q=8$ & $q=11$ & $q=14$ & $q=17$ & $q=20$ & $q=23$ \\
\hline NMHA & 42 & 107 & 163 & 233 & 297 & 366 & 419 & 492 \\
Xue's & 58 & 751 & 1850 & 3611 & 4443 & 8772 & 12,054 & 15,779 \\
\hline NO. & $q=26$ & $q=30$ & $q=34$ & $q=38$ & $q=42$ & $q=46$ & $q=50$ & $q=54$ \\
\hline NMHA & 569 & 627 & 725 & 799 & 889 & 963 & 1052 & 1138 \\
Xue's & 21,136 & 28,412 & 33,689 & 39,956 & 51,896 & 63,512 & 75,412 & 87,512 \\
\hline
\end{tabular}

The units of ATCS in Table 2 is in milliseconds. Analyzing the experimental results, it shows that the consuming time by Xue's is larger than that by NMHA, no matter what the number of destination nodes is, because the novel NMHA algorithm in this paper is devised to deal with the MCOMR problem in feasible time, different from the previous Xue's deterministic algorithm. At the same time, it could be easily found that when the value of $q$ is getting larger from 2 to 54 , the ATCS by NMHA is multiplied with the increase of destination nodes, while the ATCS by Xue's grows exponentially. The simulation experimental results also show that the NMHA algorithm has the lower time complexity level.

Following that, the performance of the NMHA algorithm would be verified, compared with the previous algorithm FMPH by the authors [20], which is finding for the approximation solution for MCOMR problem in WMNs as fast as possible. As for random networks, the topologies are generated by Waxman model. Each link of random networks has the same three weights as the RNET network. Ten topologies for random networks are generated randomly, each of which has 50 nodes or 500 nodes. On each topology, 10 routing requests are also generated at the source node. It defines the metrics, Average Weigh Occupying (AWO) to evaluate the performance. Tables 3 and 4 show the AWO of Steiner trees with different destination nodes generated via the NMHA and FMPH algorithm in the simulation experiments, respectively:

$$
\mathrm{AWO}=\frac{\text { Total weigh occupying for each Steiner tree with q destination nodes }}{\text { Numbers of running }}
$$

Table 3. Average Weigh Occupying (AWO) $\left(10^{-3}\right)$ for NMHA algorithm and Fast Minimum Path Cost Heuristic (FMPH) algorithm with 50 nodes.

\begin{tabular}{ccccccccc}
\hline NO. & $q=2$ & $q=5$ & $q=8$ & $q=11$ & $q=14$ & $q=17$ & $q=20$ & $q=23$ \\
\hline NMHA & 24 & 33 & 34 & 56 & 58 & 59 & 70 & 59 \\
FMPH & 24 & 35 & 38 & 64 & 66 & 69 & 80 & 75 \\
\hline NO. & $q=26$ & $q=29$ & $q=32$ & $q=35$ & $q=38$ & $q=42$ & $q=46$ & $q=47$ \\
\hline NMHA & 72 & 80 & 81 & 90 & 95 & 101 & 109 & 110 \\
FMPH & 85 & 98 & 105 & 103 & 117 & 124 & 134 & 136 \\
\hline
\end{tabular}


Table 4. AWO $\left(10^{-5}\right)$ for NMHA and FMPH with 500 nodes.

\begin{tabular}{ccccccccc}
\hline NO. & $q=2$ & $q=35$ & $q=70$ & $q=105$ & $q=140$ & $q=175$ & $q=210$ & $q=245$ \\
\hline NMHA & 1009 & 1490 & 1822 & 2201 & 2330 & 2474 & 2819 & 2837 \\
FMPH & 1067 & 1490 & 2230 & 2485 & 2764 & 3310 & 3545 & 3355 \\
\hline NO. & $q=270$ & $q=305$ & $q=340$ & $q=375$ & $q=410$ & $q=445$ & $q=496$ & $q=497$ \\
\hline NMHA & 3320 & 3349 & 3610 & 3535 & 3894 & 4041 & 4488 & 4492 \\
FMPH & 4128 & 4129 & 4824 & 4877 & 5139 & 5212 & 5985 & 5989 \\
\hline
\end{tabular}

The results in Table 3 show that when there are 50 nodes in the random network, no matter how many destination nodes are involved, the AWO obtained from NMHA is always less than that from FMPH. In Table 4, when the total number of nodes in the random network reaches up to 500, the outcome is still the same. It is consistent with our analysis that the NMHA algorithm presented in this paper is looking for the approximate optimal solution in feasible time, while FMPH tends to find the approximate optimal solution as soon as possible according to the time-varying characteristics of wireless networks. From the above results and analysis, it could be concluded that, with certain destination nodes, the proposed NMHA can find a better solution compared with FMPH in feasible time.

Since the approximation factor $2(1+\varepsilon)(1-1 / q)$ solely does not illustrate the quality of the proposed algorithm, the approximation ratio will be studied in the following simulations, where we pay attention to the approximation ratio as well as its distribution. The inverse of the approximation ratio (IAR) is adopted to evaluate the performance of NMHA [35]. In other words, the ratio equal to 1 means that the solution found is the optimal solution. The ratio of less than 1 means that the solution found is sub-optimal, and the ratio of its objective to the optimal solution is represented by the value of the ratio to the optimal solution, represented by the value of the ratio. The experiments results of approximation ratio for the NMHA algorithm are shown in Figure 7. Different numbers of destination nodes (from 2 to 59) were chosen each time to finish the simulation experiments, which were run to calculate the IAR ten times. From the results, it could be concluded that the lower bound of IAR is close to 0.63 , and the upper bound is very close to 1 . Over $80 \%$ of all scenarios have the approximation factor greater than 0.8 , which means that our proposed NMHA algorithm found a solution very close to the optimal multicast routing tree.

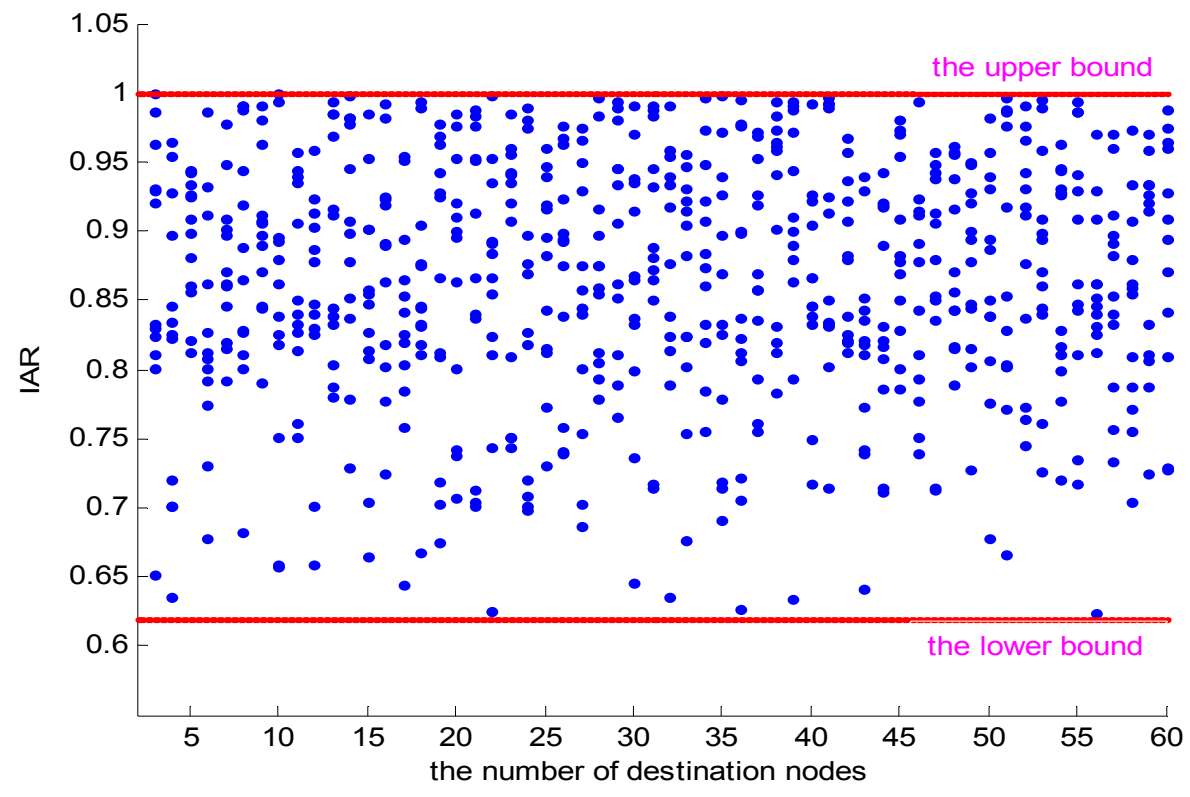

Figure 7. Experiment analysis of the approximation ratio. 


\section{Conclusions}

This paper discussed the problem of optimal constrained multicast routing in WMNs. A novel approximation algorithm for the MCOMR problem was proposed, based on the technique of auxiliary graph construction, scaling, rounding, and the MPH algorithm. The proposed algorithm can achieve lower complexity and obtain the approximation optimal multicast routing tree in feasible time. According to the experiments on the special static networks, the proposed NMHA algorithm is capable of finding the optimal (or minimum-cost) multiply constrained optimal multicast routing tree with the approximation factor of $2(1+\varepsilon)(1-1 / q)$ and the time complexity of $O\left(q m n^{2} \tau^{K-1}\right)$.

As for future research work, we plan to study more excellent solutions and faster algorithms for the MCOMR problem according to the time-varying characteristics of wireless networks based on our current research. Investigation on comparisons of the NMHA with other algorithms is also interesting.

Acknowledgments: The work described in this paper is supported by the Science and Technology Program of Guangzhou (No. 201510010192), and the Public Welfare Fund and Ability Construction Project of Guangdong Province (No. 2016A010101040), China.

Author Contributions: Weijun Yang conceived and designed the experiments, and then performed the experiments; Yuanfeng Chen analyzed the data; Weijun Yang contributed analysis tools and wrote the paper; and Yuanfeng Chen contributed to the advancement of the paper.

Conflicts of Interest: The authors declare no conflict of interest.

\section{References}

1. Zhu, Z.; Li, S.; Chen, X. Design QoS-Aware Muli-Path Provisioning Strategies for Efficient CLOUD-Assisted SVC Video Streaming to Heterogeneous Clients. IEEE Trans. Multimedia 2013, 15, 758-768.

2. Huang, J.; Huang, X.; Ma, Y. Routing with multiple quality of services constraints: An approximation perspective. J. Netw. Comput. Appl. 2012, 35, 465-475. [CrossRef]

3. Fang, X.; Yang, D.; Xue, G. MAP: Multiconstrained Anypath Routing in Wireless Mesh Networks. IEEE Trans. Mob. Comput. 2012, 12, 1893-1906. [CrossRef]

4. Lu, T.; Zhu, J. Genetic Algorithm for Energy-Efficient QoS Multicast Routing. IEEE Commun. Lett. 2013, 17, 31-34. [CrossRef]

5. Su, Y.-S.; Su, S.-L.; Li, J.-S. Joint Topology-Transparent Scheduling and QoS Routing in Ad Hoc Networks. IEEE Trans. Veh. Technol. 2014, 63, 372-389. [CrossRef]

6. Huijun, D.; Hua, Q.; Jihong, Z. QoS routing algorithm with multi-dimensions for overlay networks. China Commun. 2013, 10, 167-176. [CrossRef]

7. Xiao, Y.; Thulasiraman, K.; Fang, X.; Yang, D.; Xue, G. Computing a Most Probable Delay Constrained Path: NP-Hardness and Approximation Schemes. IEEE Trans. Comput. 2012, 61, 738-744. [CrossRef]

8. Chen, S.; Song, M.; Sahni, S. Two techniques for fast computation of constrained shortest paths. IEEE/ACM Trans. Netw. 2008, 16, 105-115. [CrossRef]

9. Huang, J.; Liu, Y. MOEAQ: A QoS-aware multicast routing algorithm for MANET. Expert Syst. Appl. 2010, 37, 1391-1399. [CrossRef]

10. Liu, L.; Song, Y.; Zhang, H.; Ma, H.; Vasilakos, A.V. Physarum Optimization: A Biology-inspired Algorithm for the Steiner Tree Problem in Networks. IEEE Trans. Comput. 2015, 64, 818-831.

11. Youssef, M.; Ibrahim, M.; Abdelatif, M.; Lin, C.; Vasilakos, A.V. Routing Metrics of Cognitive Radio Networks: A Survey. IEEE Commun. Surv. Tutor. 2014, 16, 92-109. [CrossRef]

12. Zhang, X.M.; Zhang, Y.; Yan, F.; Vasilakos, A.V. Interference-based topology control algorithm for delay-constrained mobile Ad hoc networks. IEEE Trans. Mob. Comput. 2015, 14, 742-754. [CrossRef]

13. Pei, G.; Parthasarathy, S.; Srinivasan, A.; Vullikanti, A.K.S. Approximation Algorithms for Throughput Maximization in Wireless Networks with Delay Constraints. IEEE/ACM Trans. Netw. 2013, 21, 1988-2000. [CrossRef]

14. Takahashi, H.; Matsuyama, A. An approximate solution for the Steiner problem in graphs. Math Jpn. 1980, 24, 573-577.

15. Yuan, X. Heuristic algorithms for multiconstrained quality-of-service routing. IEEE/ACM Trans. Netw. 2002, 10, 244-256. [CrossRef] 
16. Xue, G.; Sen, A.; Zhang, W.; Tang, J.; Thulasiraman, K. Finding a path subject to many additive QoS constraints. IEEE/ACM Trans. Netw. 2007, 15, 201-211. [CrossRef]

17. Xue, G.; Zhang, W.; Tang, J.; Thulasiraman, K. Polynomial time approximation algorithms for multi-constrained QoS routing. IEEE/ACM Trans. Netw. 2008, 16, 656-669.

18. Feng, G.; Korkmaz, T. A Fast Hybrid $\varepsilon$-Approximation Algorithm for Computing Constrained Shortest Paths. IEEE Commun. Lett. 2013, 17, 1471-1474. [CrossRef]

19. Szymanski, T.H. Max-Flow Min-Cost Routing in a Future-Internet with Improved QoS Guarantees. IEEE Trans. Commun. 2013, 61, 1485-1497. [CrossRef]

20. Yang, W.; Zhang, Y. A Fast Algorithm for the Optimal Constrained Path Routing in Wireless Mesh Networks. J. Commun. 2016, 11, 126-131.

21. Li, P.; Guo, S.; Yu, S.; Vasilakos, A.V. Reliable Multicast with Pipelined Network Coding Using Opportunistic Feeding and Routing. IEEE Trans. Parallel Distrib. Syst. 2014, 25, 3264-3273. [CrossRef]

22. Meng, T.; Wu, F.; Yang, Z.; Chen, G.; Vasilakos, A. Spatial Reusability-Aware Routing in Multi-Hop Wireless Networks. IEEE Trans. Comput. 2015, 65. [CrossRef]

23. Liu, J.; Wan, J.; Wang, Q.; Zeng, B.; Fang, S. A Time-recordable Cross-Layer Communication Protocol for the Positioning of Vehicular Cyber-Physical Systems. Future Gener. Comput. Syst. 2016, 56, 438-448. [CrossRef]

24. Liu, J.; Wan, J.; Wang, Q.; Li, D.; Qiao, Y.; Cai, H. A Novel Energy-saving One-Sided Synchronous Two-Way Ranging Algorithm for Vehicular Positioning. ACM/Springer Mob. Netw. Appl. 2015, 20, 661-672. [CrossRef]

25. Wan, J.; Liu, J.; Shao, A.; Vasilakos, A.V.; Imran, M.; Zhou, K. Mobile Crowd Sensing for Traffic Prediction in Internet of Vehicles. Sensors 2016, 16, 88. [CrossRef] [PubMed]

26. Alasaad, A.; Nicanfar, H.; Gopalakrishnan, S.; Leung, V.C.M. A ring-based multicast routing topology with QoS support in wireless mesh networks. Wirel. Netw. 2013, 19, 1627-1651. [CrossRef]

27. Hwang, I.S.; Nikoukar, A.; Chen, K.C.; Liem, A.T.; Lu, C.H. QoS enhancement of live IPTV using an extended real-time streaming protocol in Ethernet passive optical networks. IEEE/OSA J. Opt. Commun. Netw. 2014, 6, 695-704. [CrossRef]

28. Xiang, Z.; Tao, M.; Wang, X. Coordinated Multicast Beamforming in Multicell Networks. IEEE Trans. Wirel. Commun. 2013, 12, 12-21. [CrossRef]

29. Cao, Y.; Blostein, S.D.; Chan, W.Y. Optimization of unequal error protection rateless codes for multimedia multicasting. J. Commun. Netw. 2015, 17, 221-230. [CrossRef]

30. Afolabi, R.O.; Dadlani, A.; Kim, K. Multicast Scheduling and Resource Allocation Algorithms for OFDMA-Based Systems: A Survey. IEEE Commun. Surv. Tutor. 2013, 15, 240-254. [CrossRef]

31. Al-Fuqaha, A.; Khreishah, A.; Guizani, M.; Rayes, A.; Mohammadi, M. Toward better horizontal integration among IoT services. IEEE Commun. Mag. 2015, 53, 72-79. [CrossRef]

32. Mahboobi, B.; Mehrizi, S.; Ardebilipour, M. Multicast Relay Beamforming in CDMA Networks: Nonregenerative Approach. IEEE Commun. Lett. 2015, 19, 1418-1421. [CrossRef]

33. Bornhorst, N.; Pesavento, M.; Gershman, A.B. Distributed Beamforming for Multi-Group Multicasting Relay Networks. IEEE Trans. Signal Proc. 2012, 60, 221-232. [CrossRef]

34. Chuah, S.P.; Chen, Z.; Tan, Y.P. Energy-Efficient Resource Allocation and Scheduling for Multicast of Scalable Video Over Wireless Networks. IEEE Trans. Multimed. 2012, 14, 1324-1336. [CrossRef]

35. Quang, P.T.A.; Piamrat, K.; Singh, K.D.; Viho, C. Video Streaming over Ad-hoc Networks: A QoE-based Optimal Routing Solution. IEEE Trans. Veh. Technol. 2016. [CrossRef]

(C) 2016 by the authors; licensee MDPI, Basel, Switzerland. This article is an open access article distributed under the terms and conditions of the Creative Commons Attribution (CC-BY) license (http://creativecommons.org/licenses/by/4.0/). 